\title{
Ab initio molecular dynamics simulation of hydrogen fluoride at several thermodynamic states
}

\author{
Kreitmeir, M.; Bertagnolli, H.; Mortensen, Jens Jørgen; Parrinello, M.
}

Published in:

Journal of Chemical Physics

Link to article, DOI:

$10.1063 / 1.1539045$

Publication date:

2003

Document Version

Publisher's PDF, also known as Version of record

Link back to DTU Orbit

Citation (APA):

Kreitmeir, M., Bertagnolli, H., Mortensen, J. J., \& Parrinello, M. (2003). Ab initio molecular dynamics simulation of hydrogen fluoride at several thermodynamic states. Journal of Chemical Physics, 118(8), 3639-3645. https://doi.org/10.1063/1.1539045

\section{General rights}

Copyright and moral rights for the publications made accessible in the public portal are retained by the authors and/or other copyright owners and it is a condition of accessing publications that users recognise and abide by the legal requirements associated with these rights.

- Users may download and print one copy of any publication from the public portal for the purpose of private study or research.

- You may not further distribute the material or use it for any profit-making activity or commercial gain

- You may freely distribute the URL identifying the publication in the public portal 


\title{
Ab initio molecular dynamics simulation of hydrogen fluoride at several thermodynamic states
}

\author{
Markus Kreitmeir and Helmut Bertagnolli \\ Institut für Physikalische Chemie, Universität Stuttgart, Pfaffenwaldring 55, D-70569 Stuttgart, Germany \\ Jens Jørgen Mortensen \\ Department of Physics, Technical University of Denmark, Building 307, DK-2800 Lyngby, Denmark \\ Michele Parrinello \\ CSCS Swiss Center for Scientific Computing, Via Cantonale, CH-6928 Manno, Switzerland and Physical \\ Chemistry ETH, Hoenggerberg HCI, CH-8093 Zurich, Switzerland
}

(Received 2 October 2002; accepted 26 November 2002)

\begin{abstract}
Liquid hydrogen fluoride is a simple but interesting system for studies of the influence of hydrogen bonds on physical properties. We have performed ab initio molecular dynamics simulations of HF at several thermodynamic states, where we examine the microscopic structure of the liquid as well as its static and dynamic properties. The results obtained show good agreement with well established data, and, moreover, we were able to show significant changes within the structure depending on the system's temperature and density. (C) 2003 American Institute of Physics.
\end{abstract}

[DOI: $10.1063 / 1.1539045]$

\section{INTRODUCTION}

During the last decades the importance of hydrogen bonds with respect to the structure of matter has become more and more obvious. The intramolecular shape of biomolecules, such as proteins or other macromolecules like DNA, is strongly influenced by hydrogen bonds, but the intermolecular structure is also affected. For instance, the properties of water and alcohols are strongly determined by hydrogen bonds. As hydrogen fluoride (HF) forms the strongest hydrogen bonds known, it has become a popular model system for investigations into this specific field of interest.

Solid hydrogen fluoride is constituted of parallel zig-zag chains $^{1-3}$ and it is apparent from its properties that these chains, to a certain extent, also persist in the liquid phase. To describe liquid hydrogen fluoride, many simulations have been carried out; see e.g., Refs. 4-9.

Simulations based on effective potentials have shown a limited ability to describe the liquid structure, although they describe other properties well. Ab initio molecular dynamics simulations carried out by Röthlisberger and Parrinello, ${ }^{9}$ on the other hand, have shown that the correlation function of liquid HF at ambient conditions can be well reproduced. This simulation confirms the persistence of short linear chains in the liquid state.

Hydrogen fluoride is difficult to investigate experimentally due to its toxicity and corrosiveness. It was only in 1985 that Deraman et al. were able to perform a neutron scattering experiment at ambient conditions. Very recently, this study has been extended to a broad range of thermodynamical states by Pfleiderer $e t$ al. ${ }^{10}$ who also performed neutron diffraction experiments.

The experiment showed that there are significant changes in the pair correlation functions as pressure and temperature are varied. In the experiment atom-atom pair correlation functions were not resolved and only the scattering length weighted total atom pair correlation function $G(r)$ was determined. From these data it was inferred that the coordination number and the chain lengths decrease as a function of temperature.

In view of the force field difficulties we have undertaken an $a b$ initio study of fluid HF at three different thermodynamic states, at temperatures ranging from $373 \mathrm{~K}$ to $473 \mathrm{~K}$ and particle densities form $24 \mathrm{~nm}^{-3}$ to $19.5 \mathrm{~nm}^{-3}$ (see Table I). At state I HF is liquid, whereas states II and III are supercritical.

Our results based on the method of Car and Parrinello show a good agreement between theory and experiment also at these higher temperatures and pressures and allow us to gain a deeper insight into liquid HF behavior, confirming and substantiating the predictions of Pfleiderer et al. ${ }^{10}$

\section{DETAILS OF THE COMPUTATIONAL SCHEME}

The well-established $a b$ initio molecular dynamics method of Car and Parrinello is described in detail in a number of publications (see e.g., Ref. 11). Therefore we give only the technical details of the simulations. For hydrogen a Barth-Car-type pseudopotential was used. ${ }^{12}$ A soft pseudopotential according to the scheme of Martins and Troullier ${ }^{13}$ developed by Röthlisberger ${ }^{9}$ was chosen for fluorine. The gradient-corrected exchange correlation functional model introduced by Becke ${ }^{14}$ for the exchange and by Lee, Yang, and Parr ${ }^{15}$ for the correlation part (GC-BLYP), which proved to give good results for hydrogen fluoride, ${ }^{9}$ was used for all simulations. All calculations were performed with the program CPMD. ${ }^{16}$

The sample consisted of 54 hydrogen fluoride molecules arranged in a body centered cubic (bcc) lattice in the initial setup. This is an improbable structure for hydrogen fluoride, and it was chosen to speed up the melting of the system. All MD simulations were performed at constant energy in a pe- 
TABLE I. Phase point, temperature $T$, number density $C$, and pressure $p$ of hydrogen fluoride taken from Refs. 17 and 18. Diffraction experiments (Ref. 10) have been performed at these thermodynamic states. Values for the boiling point are given as reference.

\begin{tabular}{lccc}
\hline \hline Phase point & $T(\mathrm{~K})$ & $C / \mathrm{nm}^{-3}$ & $p / \mathrm{bar}$ \\
\hline I & 373 & 24.0 & 12.0 \\
II & 473 & 24.0 & 319.0 \\
III & 473 & 19.5 & 166.0 \\
b.p. & 292.7 & 28.7 & 1.013 \\
\hline \hline
\end{tabular}

riodically repeated cubic cell. The volume of the cell (see Table II) was chosen to represent the experimental density, taken from Refs. 17 and 18. A time step of 0.121 fs was used, a fictitious mass for the electronic degrees of freedom of $\mu=800$ a.u. and a plane-wave cutoff energy of $E_{\text {cut }}=60 \mathrm{Ry}$ was chosen. Due to the higher temperature of the states investigated, the chosen time step is smaller than the one used for the simulations at ambient conditions. ${ }^{9}$

The preparation of the system was carried out by heating the system to an average temperature of $600 \mathrm{~K}$, which was held for roughly $0.5 \mathrm{ps}$, such that the memory of the initial configuration is lost completely. Afterwards cooling was started by rescaling the velocities of the nuclei with a factor of 0.9998 per MD step until the desired temperature was reached (see Table II). After an initial equilibration time of roughly 1 ps er state, the system was sampled for several picoseconds; see also Table II. For each state, localized Wannier functions ${ }^{19-21}$ were calculated for a simulation period of approximately $1 \mathrm{ps}$.

\section{RESULTS AND DISCUSSION}

\section{A. Radial distribution functions}

The total radial distribution function $G(r)$, which is measured in neutron diffraction experiments, is given by the linear combination of the individual pair correlation functions, ${ }^{10}$

$$
G(r)=0.4966 g_{\mathrm{HF}}+0.2104 g_{\mathrm{FF}}+0.2930 g_{\mathrm{HH}} .
$$

The coefficients of the pair correlation functions come from nuclear scattering lengths.

A comparison between the $G(r)$ obtained by the present simulations and recently published data from neutron scattering ${ }^{10}$ for the three thermodynamic states investigated here is shown in Fig. 1. For reasons of clarity, only the intermolecular part of the pair correlation functions is shown throughout this article. In the same picture we show the results from previous $a b$ initio simulations as well as a force field simulation. It is seen that the latter gives a poor descrip-

TABLE II. Phase point, temperature $T$, length of the edge of the cubic simulation cell $a$, and sampling time $t_{s}$ (without preparation and equilibration of the system).

\begin{tabular}{lccc}
\hline \hline Phase point & $T / \mathrm{K}$ & $a / \AA$ & $t_{s} / \mathrm{ps}$ \\
\hline I & 373 & 13.1037 & 3.3 \\
II & 473 & 13.1037 & 5.8 \\
III & 473 & 14.0428 & 2.1 \\
\hline \hline
\end{tabular}

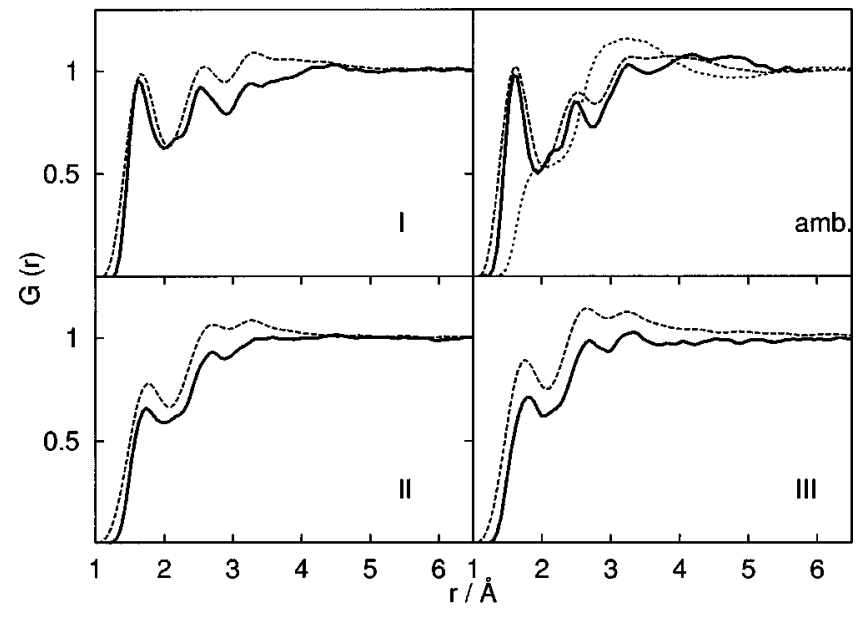

FIG. 1. Total radial distribution functions for states I, II, and III; see Table I for reference of states. Solid lines represent simulations, dashed lines scattering experiments (Ref. 10). In addition, the distribution functions for ambient conditions are shown; the broken line comes from a classical simulation (Ref. 8).

tion of the system while the $a b$ initio results faithfully reproduce the $G(r)$ and its variations with temperature and pressure.

By multiplying $G(r)$ by $4 \pi r^{2} C$ we obtain and estimate of the coordination number and the strength of the hydrogen bond (Fig. 2). From this indication a reduced probability for the formation of hydrogen bonds can be inferred. ${ }^{10}$

Having established the reliability of our model, we can now examine the origin of the $G(r)$ structures in greater detail. In fact, in contrast to experiment, we can easily obtain the three atomic pair correlation functions. These are shown in Fig. 3. As expected, the first peak in the $G(r)$ at roughly $1.7 \AA$ comes from $g_{\mathrm{HF}}(r)$ although also $g_{\mathrm{HH}}(r)$ contributes.

Since $g_{\mathrm{HF}}(r)$ reflects the formation of hydrogen bonds more closely, the decrease of the peak heights is an indication of a decrease in the number of hydrogen bonds, as postulated by Pfleiderer et al.

\section{B. Analysis of molecular associations}

The winding zig-zag chains are by far the most striking feature of solid and liquid HF. We were able to observe this characteristic property at all thermodynamic states investi-

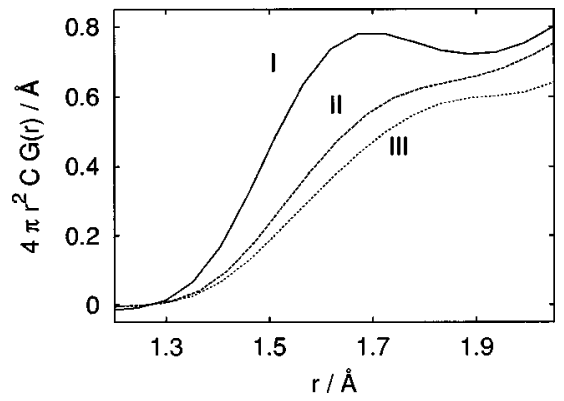

FIG. 2. Intermolecular total atom pair correlation functions weighted by $4 \pi r^{2}$ and number density $C$. Solid line represents state I, dashed line state II, and broken line state III. 


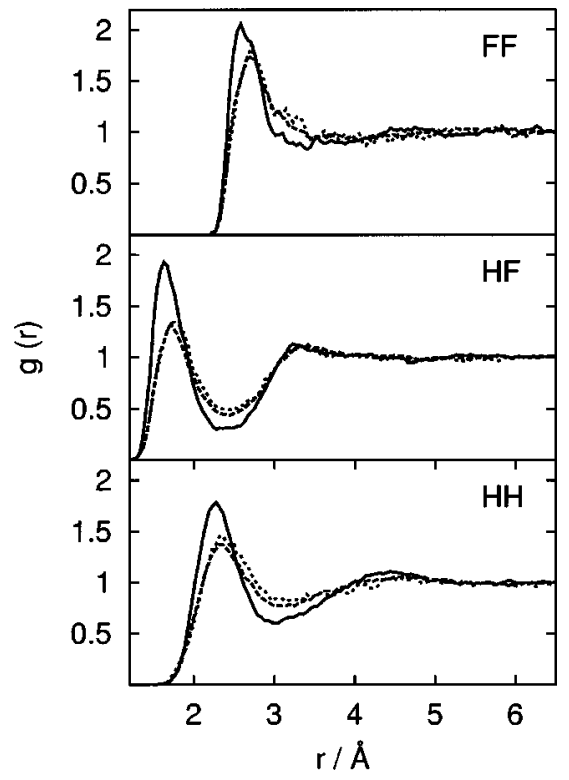

FIG. 3. Radial pair distribution functions for $g_{\mathrm{FF}}, g_{\mathrm{HF}}$, and $g_{\mathrm{HH}}$. The solid line represents state I, the dashed line state II, and the broken line state III. See Table I for reference of states.

gated. Figure 4 may serve as a qualitative impression for the bent, branched, and entwined chains of which the liquid is made up.

As HF molecules have a strong propensity to form aggregates, we performed several different approaches for an analysis of the system, enabling us to obtain a more detailed picture of liquid hydrogen fluoride, more sophisticated than interpretations of the averaged data from radial distribution functions allow. Therefore, we turn to a more molecular oriented analysis of the system.

\section{Number of hydrogen bonds per particle}

An important property of the system is the (average) number of hydrogen bonds formed per hydrogen or fluorine. This number is a function of $r_{\max }$, the maximum value where an intermolecular $\mathrm{H}$ to $\mathrm{F}$ distance is recognized as a hydrogen bond. According to this definition, no more than two hydrogen bonds per particle were observed. Figure 5 shows the total probability for the formation of one hydrogen

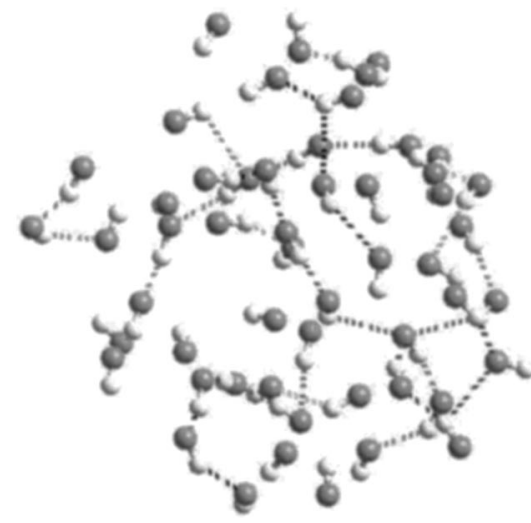

FIG. 4. Instantaneous configuration of liquid HF.

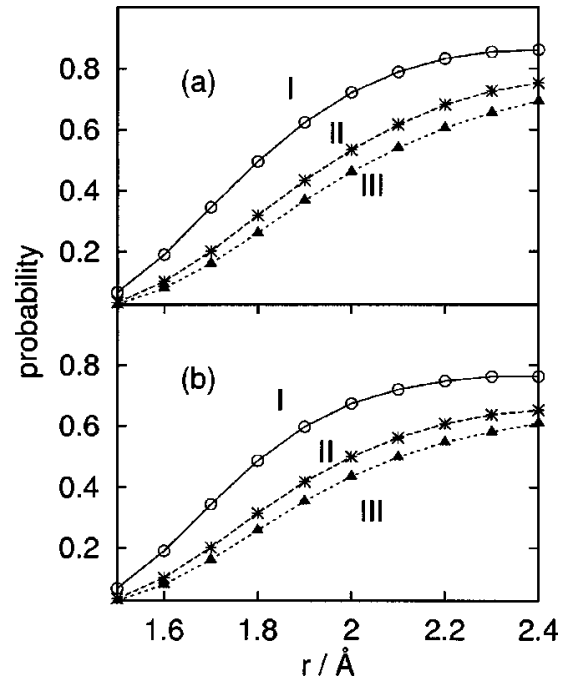

FIG. 5. Probability for forming one hydrogen bond per particle, (a) hydrogen, (b) fluorine. Circles represent state I, stars state II, and triangles state III, respectively. See Table I for reference of states.

bond per hydrogen or fluorine, whereas Fig. 6 shows the same probability for the formation of two bonds. It is clear from Fig. 5 that the probability approaches a certain level, which was reached at about $2.2 \AA$. There also the first minimum of $g_{\mathrm{HF}}$ occurs, which is equivalent to the completion of the first coordination sphere. Therefore, this distance has been chosen as reference.

Figures 5 and 6 show that, going from state I to state III, there is a decreasing probability for the formation of hydrogen bonds. The strongest decrease is caused by temperature, as can be observed from the graphs of states I and II, while changes with density (see isothermic states II and III) are less dramatic. This is in perfect agreement with the results from the discussion of the radial distribution functions in the previous section. At state I, the probability for the formation of at least one hydrogen bond, at a cutoff radius of $2.2 \AA$, is $80 \%$ for fluorine and $84 \%$ for hydrogen. For state II this probability is $66 \%$ and $69 \%$, for state III $58 \%$ and $61 \%$,

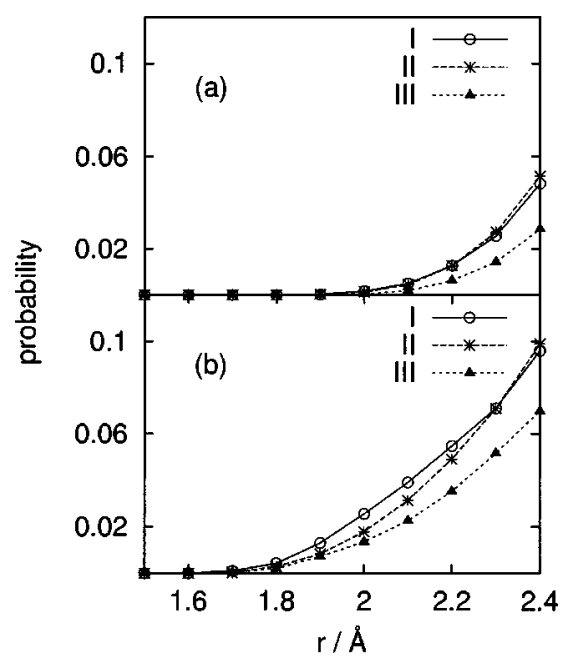

FIG. 6. Same as Fig. 5, but probability for formation of two hydrogen bonds per particle. 


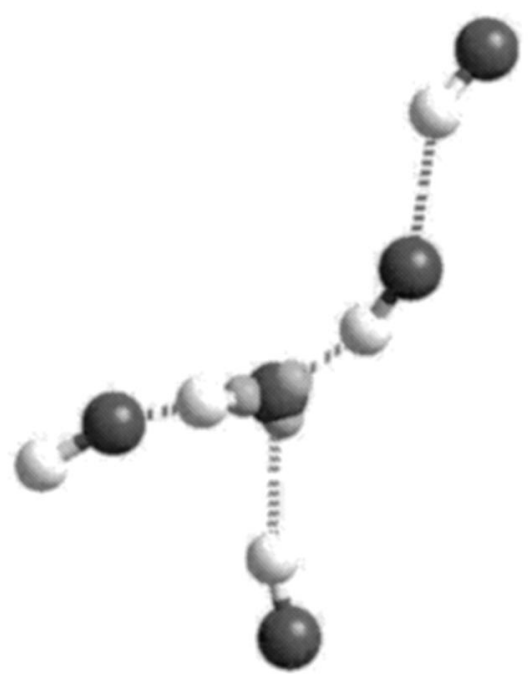

FIG. 7. A branching point of a hydrogen fluoride chain. Centers of Wannier functions of the central molecule are shown as light spheres pointing into the direction of bonded hydrogen atoms.

respectively. The decreasing discrepancy between the values from hydrogen and fluorine stem from the decreasing probability of branching points, which amounts to about $5 \%$ of the fluorine sites for states I and II and about $4 \%$ for state III. For the hydrogen sites, this probability is about $1 \%$ for all states investigated, decreasing while going from state I to III.

All states investigated show a feature that has already been observed by Röthlisberger, ${ }^{9}$ the probability of the formation of two hydrogen bonds per particle is much higher for fluorine than it is for hydrogen. This means that most of the branching points of the chains are at fluorine sites, for which the bigger size of the fluorine atom is the reason. ${ }^{9}$ In other words, the hydrogen sites are simply too small to offer space for two hydrogen bonds. Figure 7 shows a branching point of a hydrogen fluoride chain. The centers of the Wannier functions associated with the molecule, where the branch occurs, are pictured as light spheres. As one can clearly see, the function centers point in the direction of the hydrogen atoms connected via a hydrogen bond.

\section{Analysis of aggregates}

Previous molecular dynamics simulations have shown that chains are by far the most important aggregates formed by hydrogen fluoride. Therefore, we have chosen to investigate the configuration with an algorithm capable of spotting chains. The algorithm chooses a random particle and walks to both ends of the chain the particle is in. Where branches occur, the chain is considered to be continued with the strongest bond, which is formed by the bonded particle with the shortest distance. This analysis has been performed for values of $r_{\max }$ from $1.5 \AA$ to $2.4 \AA$. As is the case for the probability for forming hydrogen bonds (see previous section), the results for $2.0 \AA$ to $2.4 \AA$ are very similar. Therefore, we have again chosen a value of $r_{\max }=2.2 \AA$ as reference.

Figure 8 shows the histogram of the distribution of chain lengths determined with a cutoff radius $r_{\max }$ for the hydrogen

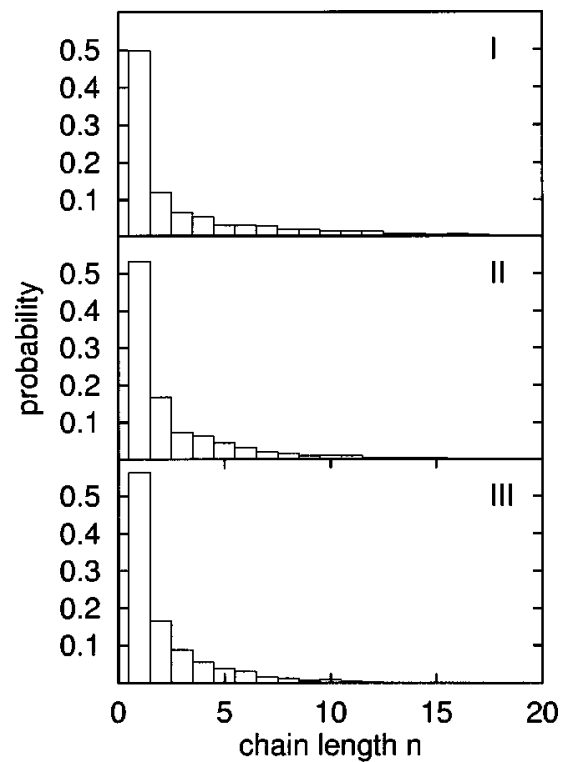

FIG. 8. Distribution of chain lengths for a cutoff distance of $r_{\max }$ of $2.2 \AA$. State I on top, state II in the middle, and state III at the bottom. See Table I for reference of states.

bonds of $2.2 \AA$. From comparison of states I and II one can see the decrease of the size of aggregates with increasing temperature. This decrease is also apparent as the density decreases (by comparison of states II and III), but the effect is less intense (see also Fig. 9 and text below).

A first look at Fig. 8 might lead to the assumption that short chains and monomers dominate the structure of liquid HF. If, however, we weigh this probability against the number $n$ of molecules present in the chain, we find a different picture. In fact the almost uniform distribution of state I becomes an exponential in the case of states II and III.

The fraction of free monomers per state is about $12 \%$, $20 \%$, and $25 \%$ for states I, II, and III, respectively. So, with

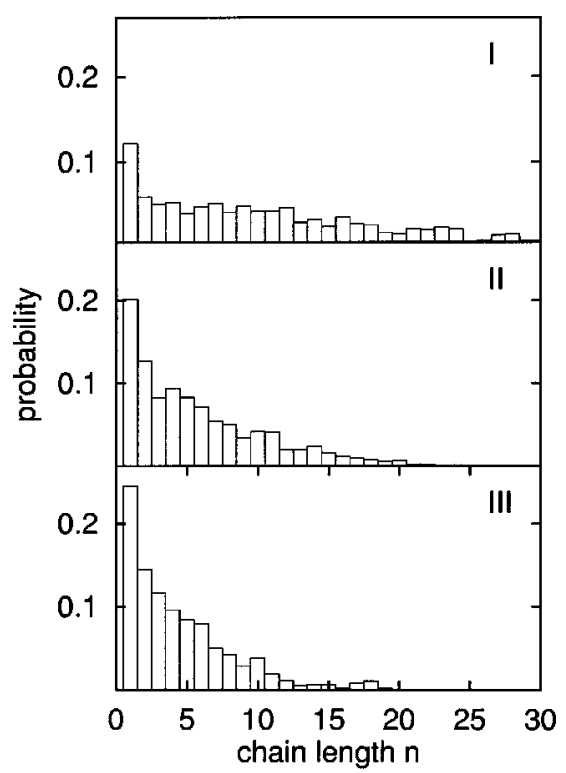

FIG. 9. Distribution of number of particles in chains of length $n$ (cutoff distance $r_{\max }$ of $2.2 \AA$ ). 


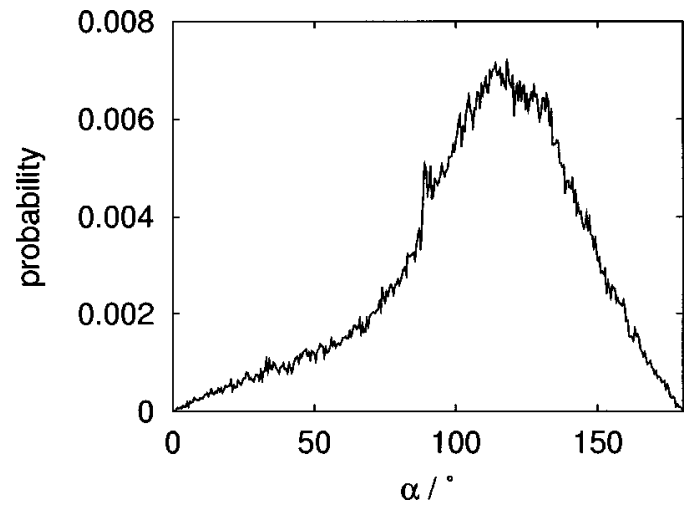

FIG. 10. Distribution of angle $\alpha$ formed by two HF molecules.

the increasing temperature as well as decreasing density at the same temperature, the occurrence of monomers is favored. The amount of the fraction of monomers for all states investigated is much higher than has been found for ambient conditions, where it is only about $6 \%{ }^{9}$

The algorithm used for analysis is, in addition, able to detect rings and apparently infinite chains. These are formed because of the periodic boundary conditions and are due to the relatively small size of the system. The number of counted infinite chains decreased strongly between state I and state III, where it was close to zero. In fact, there were three short-lived infinite chains counted during the whole sampling period. This is due to the shortening of the average chain length with temperature as well as the smaller number density of state III. The number of rings formed seems to be independent of the state considered, and their total number is very small. An inspection of the rings showed that they are often connected to a linear side chain, which makes the quantitative analysis of the ring size difficult. Nevertheless we found that, at states II and III, rings with 4 and especially 6 members are much more persistent than rings with different sizes, whereas at the liquid state I only rings with 7 and 8 members seem to be stable. This is in agreement with the liquid at ambient conditions, where rings with the same size were observed to be the most stable, too. ${ }^{9}$

Orientational correlations of the liquid are also important and interesting properties of the system. Therefore, an analysis of the angle $\alpha$ which is formed by two HF molecules forming a hydrogen bond has been performed. The distribution histogram of $\alpha$ is shown in Fig. 10 for state I (see Table I for reference of states). In contrast to the probability for forming a hydrogen bond, we did not find any significant dependence on temperature or pressure of $\alpha$, so only state I is shown. The overall average of $\alpha$ is $109^{\circ}$, whereas the maximum is between $115^{\circ}$ and $120^{\circ}$, which is in good agreement to the value obtained for the solid which is $120.1^{\circ}$ (Ref. 1).

\section{Other static properties}

The bond length for hydrogen fluoride calculated from the (averaged) distance of the bonded particles is listed in Table III. These values show good agreement with the values from scattering experiments, ${ }^{10}$ where they were deduced from the intermolecular part of the total pair distribution
TABLE III. Phase point, bond length $b$, dipole moment $\mu$, and (averaged) diffusion coefficient $D$ of hydrogen fluoride.

\begin{tabular}{lccc}
\hline \hline Phase point & $b / \AA$ & $\mu / \mathrm{D}$ & $D /\left[\mathrm{cm}^{2} / \mathrm{s}\right] 10^{-5}$ \\
\hline I & $0.955( \pm 4)$ & $2.49( \pm 5)$ & $33( \pm 5)$ \\
II & $0.950( \pm 4)$ & $2.29( \pm 5)$ & $67( \pm 5)$ \\
III & $0.949( \pm 5)$ & $2.29( \pm 4)$ & $83( \pm 5)$ \\
\hline \hline
\end{tabular}

function $G(r)$. An average, almost state independent, value of $r_{\mathrm{HF}}=0.93 \AA$ has been found there. The small decrease of the bond length with temperature shown in Table III has also been observed experimentally. There is a strong increase of the calculated bond length compared to the value for the monomer determined with the same density functional methods, where $r_{\mathrm{HF}}=0.933 \AA$ have been found. ${ }^{9}$ Experiments (Ref. 13, and references therein) obtain a distance of $r_{\mathrm{HF}}$ $=0.917 \AA$ for the HF monomer.

As localized Wannier functions were calculated for all thermodynamic states investigated, we were able to determine dipole moments for liquid hydrogen fluoride using the centers of the orbitals as centers of charge. The resulting values are also given in Table III.

The dipole moment for HF vapor is $\mu=1.91 \mathrm{D} .{ }^{22}$ Calculations for the monomer which also used DFT methods ${ }^{9}$ determined a value of $\mu=1.8 \mathrm{D}$. As has been pointed out by Briegleb, ${ }^{23}$ the dipole moment of associated HF is roughly $10 \%$ larger, e.g., $\mu=2.2 \mathrm{D}$. The values resulting from our simulations are in good agreement with these from the literature. There is a remarkable increase of the dipole moment in comparison to the monomer, as also found in water. ${ }^{24}$ Also, the increase of the dipole moment with the degree of association seems to be faithfully reproduced by the value of states II and III. Although the bond length is hardly affected by temperature and density, the electron density distribution of the molecules is. This, in turn, should affect the association of the molecules, e.g., size and kind of agglomerates formed, as could be shown in the previous section.

\section{Dynamic properties}

\section{Proton transfer and self-diffusion}

The configurations from our sample were checked for possible proton transfer processes, which could result in the formation of protonated complexes and $\mathrm{FHF}^{-}$anions. But the investigation of the covalent HF bond showed that no bond was broken or formed, and joint atoms did not rearrange during the simulation runs, although the relatively high autoionization constant of hydrogen fluoride, the sample size, and the simulation time were too small to observe the rare event of a proton transfer (see Ref. 25 for an investigation of water).

Self-diffusion coefficients $D$ were determined from the slope of the mean square displacement, which was calculated separately for hydrogen and fluorine. At all thermodynamic states the different species moved on the same time scale and diffusion coefficients were equal within the range of estimated error, which was to be expected since the system is fully molecular. The values calculated are given in Table III. The value at $373 \mathrm{~K}$ increases compared to the liquid at am- 


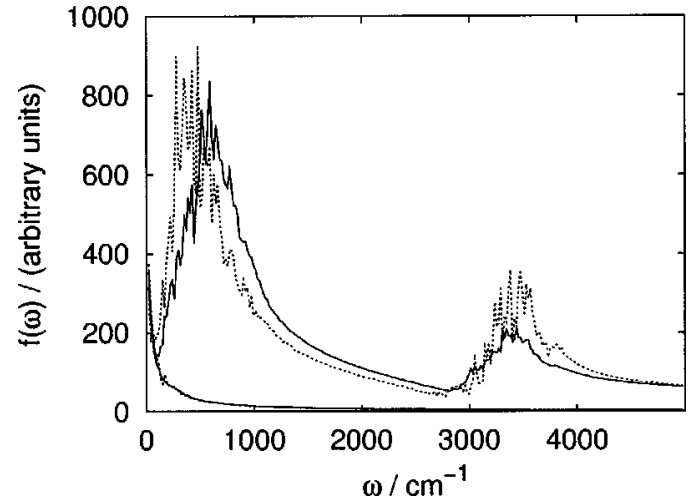

FIG. 11. Vibrational spectrum of hydrogen fluoride, contributions of hydrogen and fluorine shown separately. Solid line represents state I, broken line state III; see Table I for reference of states.

bient conditions $\left[7 \times 10^{-5} \mathrm{~cm}^{2} / \mathrm{s}\right.$ (Ref. 26)] by roughly a factor of 4. A further increase to $473 \mathrm{~K}$ approximately doubles the diffusion coefficient, and, as expected, it becomes larger still for lower densities.

\section{Vibrational spectrum}

The large mass difference between hydrogen and fluorine leads to a strong separation of high and low frequency modes. The high frequency modes are associated with hydrogen movement and the low frequencies with fluorine movement, respectively. Therefore, we show the contributions of the different atom types to the vibrational spectrum for hydrogen and fluorine separately; see Fig. 11 for spectra of states I and III (state II omitted for reasons of clarity). The spectrum was obtained by a Fourier transformation of the normalized velocity autocorrelation functions $\psi(t)$, where the hydrogen motion can be observed as beats, as can be seen from Fig. 12 for state III.

The fast decaying fluorine spectrum is mostly associated with intermolecular processes, whereas the hydrogen spectrum shows a more detailed structure. Two features can clearly be separated for all investigated states, a high frequency mode at around $3300 \mathrm{~cm}^{-1}$ and a band at about $500-$ $600 \mathrm{~cm}^{-1}$. The band associated with the intramolecular stretching mode, the high frequency mode, does not change very much in position and shape, and it is in good agreement with Raman and IR experiments, ${ }^{27}$ which show a broad absorption between $3000 \mathrm{~cm}^{-1}$ and $3600 \mathrm{~cm}^{-1}$ centered at

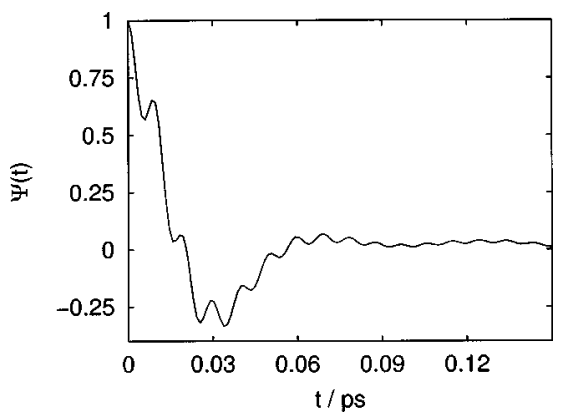

FIG. 12. Detail of the normalized velocity auto correlation function for hydrogen at state III; see Table I for reference of states.
$3400 \mathrm{~cm}^{-1}$. This once more shows good agreement, where molecular properties of HF are concerned. These properties do not seem to be affected very much by temperature.

Otherwise, the center of the second absorption band moves to lower frequencies. As this mode is associated with intermolecular $\mathrm{HF} \cdots \mathrm{H}$ stretching modes, one can deduce the decrease of the related force constant. This is in perfect agreement with the conclusion from previous sections, that the hydrogen bonds in hydrogen fluoride become weaker at higher temperature.

\section{SUMMARY AND CONCLUSIONS}

$A b$ initio molecular dynamics simulations of hydrogen fluoride have been performed for several thermodynamic states, including supercritical states. The structural data deduced from these simulations have shown good agreement with experimental results, especially recently published data from neutron scattering experiments. The intermolecular structure, which is characterized predominantly by zig-zag chains, could be reproduced with good accuracy. We could show that the average length of these chains decreases with temperature as well as density at isothermic states. The same dependence was observed for the probability for the formation of hydrogen bonds.

In contrast to the changes in the intermolecular structure, the molecular properties of HF are much less affected by temperature and density and are in good agreement with experimental data and simulations of liquid hydrogen fluoride at ambient conditions.

The data presented show once more that ab initio molecular dynamics simulations based on density functional theory are a powerful tool for the investigation of hydrogen bonded systems, also over a broad range of thermodynamic states.

\section{ACKNOWLEDGMENTS}

We would like to thank A. P. Seitsonen and C. J. Mundy for useful discussions and the HLRS at Stuttgart for providing financial support and computers. Moreover, we gratefully acknowledge the Python development team, as all data analysis was performed with this computer language.

\footnotetext{
${ }^{1}$ M. Atoji and W. Lipscomb, Acta Crystallogr. 7, 173 (1954).

${ }^{2}$ M. Johnson, E. Sandor, and E. Arzi, Acta Crystallogr., Sect. B: Struct. Crystallogr. Cryst. Chem. 31, 1998 (1975).

${ }^{3}$ D. Pinnick, A. Katz, and R. Hanson, Phys. Rev. B 39, 8677 (1989)

${ }^{4}$ M. Klein and I. McDonald, J. Chem. Phys. 71, 298 (1979).

${ }^{5}$ M. Cournoyer and W. Jorgensen, Mol. Phys. 51, 119 (1983).

${ }^{6}$ P. Jedlovszky and R. Vallauri, Mol. Phys. 93, 15 (1998).

${ }^{7}$ P. Jedlovszky and R. Vallauri, J. Chem. Phys. 107, 10166 (1997).

${ }^{8}$ R. D. Valle and D. Gazillo, Phys. Rev. B 59, 13699 (1999).

${ }^{9}$ U. Röthlisberger and M. Parrinello, J. Chem. Phys. 106, 4658 (1997).

${ }^{10}$ T. Pfleiderer, I. Waldner, H. Bertagnolli, K. Tödheide, and H. Fischer, J. Chem. Phys. 113, 3690 (2000).

${ }^{11}$ D. Remler and P. Madden, Mol. Phys. 70, 921 (1990).

${ }^{12}$ K. Laasonen, M. Sprik, M. Parrinello, and R. Car, J. Chem. Phys. 99, 9080 (1993).

${ }^{13}$ N. Troullier and J. Martins, Phys. Rev. B 43, 1993 (1991).

${ }^{14}$ A. Becke, Phys. Rev. A 38, 3098 (1988).

${ }^{15}$ C. Lee, W. Yang, and R. Parr, Phys. Rev. B 37, 785 (1988).

${ }^{16} \mathrm{~J}$. Hutter et al., CPMD (Copyright IBM Zurich Research Laboratory and MPI für Festkörperforschung, 1995-2001).
} 
${ }^{17}$ E. U. Franck and W. Spalhoff, Z. Elektrochem. 61, 348 (1957).

${ }^{18}$ E. U. Franck, G. Weigand, and R. Gerhardt, J. Supercrit. Fluids 15, 127 (1999).

${ }^{19}$ N. Marzari and D. Vanderbilt, Phys. Rev. B 56, 12847 (1997)

${ }^{20}$ P. L. Silvestrelli, N. Marzari, D. Vanderbilt, and M. Parrinello, Solid State Commun. 107, 7 (1998).

${ }^{21}$ G. Berghold, C. Mundy, A. Romero, J. Hunter, and M. Parrinello, Phys. Rev. B 61, 10040 (1999).
${ }^{22}$ N. Hannay and C. Smith, J. Am. Chem. Soc. 68, 171 (1946).

${ }^{23}$ G. Briegleb, Z. Elektrochem. Angew. Phys. Chem. 50, 35 (1944).

${ }^{24}$ P. Silvestrelli and M. Parrinello, Phys. Rev. Lett. 82, 3308 (1999).

${ }^{25}$ P. Geissler, C. Dellago, D. Chandler, J. Hutter, M. Parrinello, and R. Car, Science 291, 2121 (2001).

${ }^{26}$ R. Cole, J. Chem. Phys. 59, 1545 (1973).

${ }^{27}$ I. Sheft and A. Perkins, J. Inorg. Nucl. Chem. 38, 665 (1976). 